\title{
Functionality and spatial relations in memory and language
}

\author{
GABRIEL A. RADVANSKY and DAVID E. COPELAND \\ University of Notre Dame, Notre Dame, Indiana
}

\begin{abstract}
We examined whether the functionality of spatial relations affects the construction and memory of information in situation models. A functional relationship involves the interaction of entities that is implied by either typical use or contextual demands. Previous research has shown that spatial relations are less likely to be encoded during comprehension unless there is extensive prior knowledge, explicit instructions to attend to spatial information, or a clear emphasis on spatial information. If the construction of a situation model is guided by a need to understand the functional structure of a situation, then functional spatial relations should be more likely to be encoded. The results of our study showed that sentences with functional spatial relations were read faster and remembered better in both recall and recognition tests than sentences with nonfunctional spatial relations.
\end{abstract}

A situation model is a mental representation of a specific situation (Johnson-Laird, 1983; van Dijk \& Kintsch, 1983; Zwaan \& Radvansky, 1998). In text comprehension, situation models represent the described situation, not the text itself (Glenberg, Meyer, \& Lindem, 1987). Thus, these representations serve as working models of the world. The organization of information in situation models not only is relevant for comprehension, but also has implications for the later memory of that information (Radvansky \& Zacks, 1997). This is important because information that is comprehended is relatively valueless unless it can be usefully retained and retrieved over time. The present study assessed whether the functionality of relational information affects its encoding and memory in a situation model. The particular focus was on spatial relations because they are a situational component that is relatively easy to test in an objective fashion and because spatial information is one of the more heavily studied aspects of situation models.

\section{Functionality}

For our purposes, the term functionality refers to the actual or likely interaction between two or more entities in a situation in which the state of one entity would be affected by a second entity in a meaningful way. There are several aspects of this description that warrant elaboration. First, there is the idea of an interaction. There is some involvement of the entities with one another in a functional

Portions of this research were presented at the 1999 meeting of the Midwestern Psychological Association in Chicago. We thank Laura Carlson-Radvansky and Rolf Zwaan for their helpful comments during the course of the project, and Kelly Crowhurst, Jackie Curiel, and Bill Maurer for their assistance in collecting the data. Correspondence should be addressed to G. A. Radvansky, Department of Psychology, University of Notre Dame, Notre Dame, IN 46556 (e-mail: gabriel.a.radvansky. 1@ nd.edu). relation. That is, each of the entities plays some role in the situation. A second aspect is the idea that this interaction may actually occur. Alternatively, it may also be that the interaction is likely, and possible, but has not yet happened (or may never actually take place). Third, this (potential) interaction is not neutral. At a minimum, this interaction, if it were to occur, would result in at least one of the entities being changed in some way, such as getting wet, acquiring knowledge, having a fender dented, and so on.

The interaction and any potential change should be meaningful to the comprehender, thus introducing an element of subjectivity. The likelihood of a relation being viewed as functional is on a continuum. Some are more objectively functional, such as a physical interaction (e.g., if one car collides with another). Other relations may be viewed as important by only a few people. For example, when Bobby walks into the school dance, most people may not notice it except Suzie, who is madly in love with him. A person's experience of the world is meaningfully influenced by the functional relations they identify and encode. These functional relations help a person to decide what aspects of the world are important and to understand the situation appropriately. For example, if a person reads a description of a house, the points of the description he/she remembers may vary as a function of whether he/she is a prospective home buyer or a burglar (Anderson \& Pichert, 1978).

Often, a functional relation is a typical one, such as having a hammer above a nail. However, functionality can be context driven. For example, imagine a situation in which a person needs to fan a campfire, but none of the typical flane-fanning objects are around (e.g., a bellows or a newspaper). There is no typical functional relation between rocks and fires. However, if the rock is a fairly good-sized piece of shale, and because shale is flat and relatively lightweight, it can be used to fan the flames. This establishes 
a functional relation between the rock and the fire. The idea that functional relations can be influenced by characteristics of entities is similar to the idea that entity characteristics can enable causal event sequences (Trabasso, van den Broek, \& Suh, 1989) or have affordances that influence the role the entities play in a situation (Glenberg, 1997).

The functionality hypothesis is consistent with $\mathrm{Mi}$ chotte's (1963) idea that the knowledge of what objects are for and how they interact with each other provides the basis for conceptions of spatial arrangements. Michotte's idea is based on what entities do to one another: "Functional relations, then, constitute the essential fabric of the phenomenal world; they must be considered as a highly important factor in the adaptation of activities to their environment ... It is these relations which give the things around us their significance, since it is by coming to know what things do that we learn what they are. What they are for us is much more than their shape, their size, and their colour; it is above all what they are capable of doing, or what can be done by means of them" (p. 4).

Although derived to explain perceptual experience, this conception of functional relations is important for understanding situation models. Functional relations are at the heart of understanding what a situation is, how it develops, how it relates to other situations, and what it means to us. The concept of functionality is related to the idea that causality and intentionality are important to model processing (Zwaan \& Radvansky, 1998). Often, a functional relation implies an impending, ongoing, or prior causal relation between two or more entities. Evidence suggests that people monitor causal and intentional information during comprehension (e.g., Lutz \& Radvansky, 1997). Also, causal information is rated as more important (van den Broek, 1988) and is better remembered than information that is not part of the causal structure (Myers, Shinjo, \& Duffy, 1987).

Using our conception of functionality, we can make some assumptions. First, a functional relation should be easier to process because it is easier to integrate into the preceding context. Functional relations help define the structure of the situation. Second, information that is more peripheral is more difficult to integrate because it is unclear how it relates to other aspects of the model. Third, functional information should be more integrated into a situation model. This occurs because functional information is likely to be linked with more parts of the situation than is nonfunctional information. Thus, functional information should be better remembered.

With regard to spatial relations, a functional spatial relation allows two or more entities to interact (whether they actually do or not). This point was demonstrated in a study by Carlson-Radvansky and Radvansky (1996) that had people view a set of pictures. One task was to rate the acceptability of sentences describing spatial relations between object pairs (e.g., "The mail carrier is in front of the mailbox"). Another task was to complete sentences with the missing spatial terms (e.g., "The mail carrier is the mailbox"). The results showed that functionality influenced spatial information use. For objects in a functional relation (e.g., the mail carrier facing the mailbox), object-centered relations were preferred, consistent with the idea that people focused on the interaction of the objects. However, for objects in a nonfunctional relation (e.g., the mail carrier facing away from the mailbox), viewer- or environment-center relations were preferred, consistent with the idea that people did not consider the interaction of the objects (for a similar discussion of the importance of functionality and spatial relations, see Langston, Kramer, \& Glenberg, 1998). More recent work by Carlson-Radvansky, Covey, and Lattanzi (1999) has shown that functionality is also important in defining spatial relations (see also Carlson-Radvansky \& Tang, 2000; Coventry, Carmichael, \& Garrod, 1994; Coventry $\&$ Mather, in press).

\section{Spatial Information and Situation Models}

Our focus on spatial information is important because the situation model literature has somewhat differing results on the role of spatial knowledge in model use. Some studies seem to suggest that spatial information plays a central role. It has been found that models are easier to construct when the information clearly refers to a common spatial structure (Ehrlich \& Johnson-Laird, 1982). Also, information availability may be influenced by its relative orientation to a central entity, such as a protagonist (Franklin \& Tversky, 1990). In addition, shifts in spatial location may result in an increased processing effort (Zwaan, Radvansky, Hilliard, \& Curiel, 1998). When spatial shifts cause two entities to be separated, information about the more distant entity is less available (Glenberg et al., 1987; Morrow, Greenspan, \& Bower, 1987; Rinck $\&$ Bower, 1995). Furthermore, when entities are spatially separated and information is encountered that suggests that they are interacting as if they were in a common space, people may notice these inconsistencies (de Vega, 1995; O'Brien \& Albrecht, 1992).

Spatial information in a model also has consequences for memory retrieval, such as whether a memory probe describes events that have the same spatial arrangement as the original information (Bransford, Barclay, \& Franks, 1972; Garnham, 1981; Radvansky, Gerard, Zacks, \& Hasher, 1990). Moreover, this use of models to verify spatial information is more likely when information can initially be interpreted as referring to a single situation than otherwise (Baguley \& Payne, 1999; Mani \& JohnsonLaird, 1982). In addition, information that refers to a common location can be integrated into a single model, reducing retrieval interference (Radvansky, 1998, 1999; Radvansky, Spieler, \& Zacks, 1993; Radvansky \& Zacks, 1991; Radvansky, Zacks, \& Hasher, 1996). Finally, when asked to sort information from a previously read text, people may segregate information from different spatial locations (Zwaan, Langston, \& Graesser, 1995).

However, spatial effects are not consistently observed. For example, model updating does not always reflect spa- 
tial characteristics (Wilson, Rinck, McNamara, Bower, \& Morrow, 1993; but see Rinck \& Bower, 1995). Zwaan and van Oostendorp (1993) found that readers of naturalistic texts are often unable to report spatial relations that were explicitly mentioned. Also, reading times are reliably affected by shifts along some situation components, such as time and causality, but not space (Zwaan, Magliano, \& Graesser, 1995; Zwaan et al., 1998). In a recent study, Langston et al. (1998) failed to find an influence of spatial relations on the ability to notice that items mentioned in a text were spatially close. If people routinely monitored spatial information, then items that are spatially closer should be more available. Finally, in memory retrieval, people may favor another organizational basis (Radvansky, 1998; Radvansky et al., 1993), such as orienting around the person when a locationbased organization is implausible (e.g., having three people in a telephone booth). Also, people may use multiple organizational bases involving important situational components, such as people, in addition to space when these alternatives have similar plausibility (Radvansky et al., 1993; Taylor \& Tversky, 1997).

Why are spatial influences sometimes clearly observed in situation models and other times not? One idea is that when spatial effects are observed, the focus is almost exclusively on spatial information (e.g., Bransford et al., 1972; Franklin \& Tversky, 1990; Radvansky \& Zacks, 1991). The information is not very complex in terms of the number of situation components involved. This is especially true when there is little other information competing with the spatial information (such as a protagonist's goals). In contrast, in many cases where spatial influences are not observed, there is a lot of information about other aspects of a situation, such as causality and temporality (see, e.g., Zwaan, Magliano, \& Graesser, 1995). This increased complexity makes it less likely that people will focus on and meaningfully encode the spatial information.

Another issue is the idea that models require some effort to construct. If space does not play a central role in the text or task, people are less likely to encode or infer this information (Zwaan \& van Oostendorp, 1993). However, if there is adequate prior knowledge, then less effort is needed and spatial effects are observed (Zwaan et al., 1998). Also, if people are instructed to encode spatial information, its use can be more clearly observed than it would be without such instructions, albeit with increased processing effort (Zwaan \& van Oostendorp, 1993).

While these ideas are plausible, they leave out some important findings. For example, Langston et al. (1998) used information that focused entirely on spatial information. Still, spatial effects were not reliably observed. Also, studies that involve noticing spatial incongruities (e.g., O'Brien \& Albrecht, 1992) show spatial effects, even though the texts involved complex information about other situational components, did not require extensive background knowledge, and did not excessively direct readers' attention to the spatial information. Thus, some principle is needed to predict when spatial information is used in model processing. The present study provides some insight into this issue by focusing on functionality and spatial relations.

\section{Present Study}

The aim of this experiment was to test the functionality hypothesis. If functionality is important, then people should be more likely to incorporate relations that are more functional into a situation model due to a greater ease of integrating them into the model. In general, information that is consistent with what has occurred previously is read faster than information that does not clearly fit the current situation in the text (Zwaan, Magliano, \& Graesser, 1995). This should be reflected as faster reading times for functional sentences than nonfunctional ones. Furthermore, because that information is more likely to be encoded, it is more likely to be remembered later. As such, functional relations should be recalled and recognized more often than nonfunctional ones.

\section{METHOD}

\section{Participants}

Forty-eight students from the University of Notre Dame, all native English speakers, received partial course credit in exchange for participation. Three additional subjects were replaced for having more than $10 \%$ errors on the comprehension questions.

\section{Materials}

The 16 stories that were used were written so that each contained a critical sentence that conveyed a functional spatial relation in one context, but a nonfunctional relation in the other context. For example, in the story from Appendix A, the critical sentence appeared after the functional context of "A steady rain began to pour from the sky." The nonfunctional context was "The clouds were blocking out the moonlight, making the night darker. He needed some light so he could read his map." For each participant, half of the stories contained the functional context, and half contained the nonfunctional context. Both versions of each of the texts were rotated across participants. These stories were 13 to 17 sentences long. In addition there were two practice stories that were used to familiarize people with the experimental procedure.

In addition, two comprehension questions were created for each story. For the story in Appendix A, the questions were "Was it raining?" and "Was David's wallet stolen?" These questions were used to ensure that the participants had read the stories. Overall, half of the questions were true and half were not. None of these questions asked about information in the critical sentences.

\section{Procedure}

Reading. People were tested individually. The texts were presented on a PC-compatible computer. The text was white on a black background in 40-column mode. People read the two practice stories first, followed by the experimental texts. The experimental stories were read in a different random order for each person. Reading was self-paced. The texts were presented one clause at a time. Each of the critical items was a single-clause sentence. After reading a clause, the participant pressed the space bar with the left hand and the next clause appeared. The computer recorded reading times. After 
each story, two comprehension questions were answered by clicking one of the two buttons on the mouse with the right hand. The left mouse button was pressed for "yes," and the right mouse button for "no."

Recall. After reading all of the stories, people were presented with the first two sentences of each story and were asked to recall the remainder, reporting as much information as they could remember. Each story recall was made on a separate sheet of paper. These data were later scored on whether the critical spatial relation was recalled.

Recognition. After the recall test, people were given a recognition test. For this test, four versions of each critical sentence were presented. One of these was the original functional version (e.g., "David was standing below an old bridge"). There were three distractor versions. The nonfunctional-object version altered the object from the critical sentence (e.g., "David was standing below a lamppost"). The nonfunctional-spatial term version altered the spatial relation (e.g., "David was standing in front of an old bridge"). Finally, the nonfunctional-both version altered both of these (e.g., "David was standing in front of a lamppost"). Participants made their responses by circling the letter corresponding to the sentence they remembered reading earlier.

Analysis. Reading times were converted to milliseconds per syllable. The slowest and fastest reading times per participant per condition were excluded to remove any outliers from the analysis. For the recall task, a liberal scoring criterion was adopted. Critical items were judged to be correctly recalled if the spatial relation was expressed unambiguously. That is, the critical sentence did not have to be recalled verbatim, as long as it conveyed a spatial relation and could not be confused with another version of that critical sentence. For example, a recall of "David was facing the bridge" would be considered correct for the sentence "David was standing in front of an old bridge." However, "David was at the bridge" would be considered incorrect. Analyses done with participants as a random factor are denoted with a subscript 1 , and those done with items as a random factor are denoted with a subscript 2 . A criterion of $p<.05$ was used for all analyses.

\section{RESULTS AND DISCUSSION}

Results from the experiment are summarized in Table 1. The critical sentences were read faster $\left[t_{1}(47)=3.62\right.$, $\left.t_{2}(15)=4.02\right]$, recalled better $\left[t_{1}(47)=2.27, t_{2}(15)=\right.$ 2.37], and recognized better $\left[t_{1}(47)=4.76, t_{2}(15)=2.92\right]$ in the functional context than in the nonfunctional context. This is consistent with the functionality hypothesis. The nature of the spatial relation affects how it is processed. This is consistent with the idea that functional spatial relations were easier to integrate into the situation model, and is consistent with work showing that information that is consistent with the current situation model is easier to process (e.g., Zwaan, Magliano, \& Graesser, 1995). Although people were more likely to encode a functional than a nonfunctional relation, this does not mean that they never encode nonfunctional relations, as can be seen by the performance in the nonfunctional con-

Table 1

Reading Times (in Milliseconds/Syllable), Recall, and Recognition for the Critical Sentences

\begin{tabular}{|c|c|c|c|c|c|c|}
\hline \multirow[b]{2}{*}{ Condition } & \multicolumn{2}{|c|}{ Reading Time } & \multicolumn{2}{|c|}{ Recall } & \multicolumn{2}{|c|}{ Recognition } \\
\hline & $M$ & $S E$ & $M$ & $S E$ & $M$ & $S E$ \\
\hline inctiona & 175 & 5 & $46 \%$ & 3 & $87 \%$ & 2 \\
\hline Nonfunctional & 200 & 8 & $39 \%$ & 3 & $74 \%$ & 2 \\
\hline
\end{tabular}

dition. It should also be noted that this superior memory for functional spatial relations is true even though people spent more time processing the information in the nonfunctional versions according to the reading time data. This is consistent with the idea that functional spatial relations are more likely to be incorporated into the situation model and stored in memory.

These results have also been replicated in three other experiments conducted in our laboratory using somewhat different methodologies. These experiments are described briefly in Appendix B. These data lend further support to the influence of functionality on processing and the conclusions that we have drawn from the main experiment.

\section{DISCUSSION}

Our findings support three main conclusions. First, consistent with other research (Zwaan \& van Oostendorp, 1993), it appears that spatial relations are not consistently encoded and stored in situation models. Situation models are complex representations and there is some flexibility in the degree to which various components are emphasized (Radvansky, 1998; Zwaan \& Radvansky, 1998). The present study suggests that, while spatial information can influence situation model processing, there are limits to the circumstances in which people employ this type of knowledge.

Second, people are more likely to encode and store spatial relations in a situation model the more functional they are. This is consistent with the idea that functionality is important for processing spatial relations (e.g., CarlsonRadvansky \& Radvansky, 1996). People are concerned not only with how entities are positioned with respect to one another, but also with how they interact (or do not). Because situation models are often incomplete (JohnsonLaird, 1983), the information most likely to be encoded is that which defines how the entities are interacting.

Finally, functionality is affected by context. In general, situation models are defined by the structure of situations as they would exist in the world and how they operate (Johnson-Laird, 1983). These operations are defined both by how the person interacts with the situation and by how the other components of the situation interact with one another. Thus, in memory and comprehension, the provided complex of information can have consequences that are not immediately derivable from the properties of the individual components. There are qualities of cognition that are only observable when the whole is considered.

The functionality hypothesis can be applied to a broader range of research on spatial situation models to provide some insight into why influences of spatial information are sometimes clearly observed, and other times not. In this section we will speculate on how functionality may be involved. Obviously, more direct study is needed to follow up on these ideas.

For studies in which the spatial relations were the focus (e.g., Bransford et al., 1972; Ehrlich \& Johnson-Laird, 1982; Radvansky \& Zacks, 1991), an explicit understand- 
ing of the spatial structure of the situation is needed to perform the task, and thus serves as functional information. When there is extensive prior knowledge about an area or region, subsequent information about spatial shifts is more meaningful (e.g., Morrow et al., 1987; Rinck \& Bower, 1995; Zwaan et al., 1998). A person can understand how entities, such as a protagonist, can interact (or not) with other entities in the story on the basis of their spatial relation. Also, if people are instructed to encode spatial information (Zwaan \& van Oostendorp, 1993), the task makes the information functionally important, making it more likely to be encoded.

The functionality hypothesis is also consistent with studies where spatial information use in situation models was not observed. For example, in the Wilson et al. (1993) study, because the probed-for objects were not related to the protagonist, there was little reason to relate their location to the protagonist's in the model. The participant needed only to refer to a general mental map created prior to reading. In studies using more complex and naturalistic texts (Zwaan \& van Oostendorp, 1993), it is unlikely that the described spatial arrangements of entities were important to understanding the major functional interactions among them. Often, the dynamics of a situation are not spatially oriented. However, spatial information may be encoded to some degree even in these cases (Zwaan, Magliano, \& Graesser, 1995). A similar point can be made for the Langston et al. (1998) study. Although those texts focused on spatial information, there was no reason to consider how the objects were interacting with one another in that space. As such, the spatial relations were not functional, so they were not elaborated on and spatial noticing effects were not observed. Finally, spatial location is not used to organize information when such an organization would violate social norms about the number of people that can occupy small locations, such as a telephone booth (Radvansky, 1998; Radvansky et al., 1993). Thus, how people and spatial locations function in the world influences how people mentally represent this information.

In general, it appears that the functionality of spatial relations plays an important role in the degree to which they are encoded and stored in situation models in memory. This was observed across a number of cognitive measures, namely reading times, recall rates, and recognition accuracy. It seems likely that functionality can be generalized and has a pervasive role in the processing of situation model information.

\section{REFERENCES}

ANDERSON, R. C., \& Pichert, J. W. (1978). Recall of previously unrecallable information following a shift in perspective. Journal of Verbal Learning \& Verhal Behavior, 17, 1-12.

Baguley, T., \& Payne. S. J. (1999). Recognition memory for sentences from spatial descriptions: A test of the episodic construction trace hypothesis. Memory \& Cognition, 27, 962-973.

Bransford, J. D., Barclay, J. R., \& Franks, J. J. (1972). Sentence memory: A constructive versus interpretive approach. Cognitive Psychologv, 3, 193-209.
Carlson-Radvansky, L. A., Covey, E. S., \& LatTanzi, K. M. (1999). "What" effects on "where": Functional influences on spatial relations. Psychological Science, 10, 516-521

Carlson-Radvansky, L. A., \& Radvansky, G. A. (1996). The influence of functional relations on spatial term selection. Psychological Science, 7, 56-60.

Carlson-Radvansky, L. A., \& Tang, Z. (2000). Functional influences on orienting a reference frame. Memory \& Cognition, 28, 812-820.

Coventry, K. R., Carmichael, R., \& Garrod, S. C. (1994). Spatial prepositions, object-specific function, and task requirements. Journal of Semantics, 11, 289-309.

Coventry, K. R., \& Mather, G. (in press). The real story of "over." In P. Oliver \& W. Mass (Eds.), Vision and language. New York: Springer-Verlag.

DE VEGA, M. ( 1995). Backward updating of mental models during continuous reading of narratives. Journal of Experimental Psychology: Learning, Memory, \& Cognition, 21, 373-385.

EHRLICH, K., \& JohNSON-LAIRD, P. N. (1982). Spatial descriptions and referential continuity. Journal of Verbal Learning \& Verbal Behavior, 21, 296-306.

FranKLIN, N., \& TVERSKy, B. (1990). Searching imagined environments. Journal of Experimental Psychology: General, 119, 63-76.

Garnham, A. (1981). Mental models as representations of text. Memory \& Cognition, 9, 560-565.

GLENBERG, A. M. (1997). What memory is for. Behavioral \& Brain Sciences, 20, 1-55.

GlenberG, A. M., Meyer, M., \& Lindem, K. (1987). Mental models contribute to foregrounding during text comprehension. Journal of Memory \& Language, 26, 69-83.

JOHNSON-LAIRD, P. N. (1983). Mental models: Towards a cognitive science of language, inference, and consciousness. Cambridge, MA: Harvard University Press.

Langston, W., Kramer, D. C., \& Glenberg, A. M. (1998). The representation of space in mental models derived from text. Memory \& Cognition, 26, 247-262.

LuTZ, M. F., \& RadVansky, G. A. (1997). The fate of completed goal information in narrative comprehension. Journal of Memory \& Language, 36, 293-310.

MANI, K., \& JoHNSON-LAiRD, P. N. ( 1982 ). The mental representation of spatial descriptions. Memory \& Cognition, 10, 181-187.

MichotTE, A. (1963). The perception of causality (T. R. Miles, Trans.). London: Methuen. (Original work published 1946)

Morrow, D. G., Greenspan, S. L., \& Bower, G. H. (1987). Accessibility and situation models in comprehension. Journal of Memory \& Language, 26, 165-187.

Myers, J. L., Shinjo, M., \& Duffy, S. A. (1987). Degree of causal relatedness and memory. Journal of Memory \& Language, 26, 453-465.

O'BRIEN, E. J., \& AlbReCht, J. E. (1992). Comprehension strategies in the development of a mental model. Journal of Experimental Psychology: Learning, Memory, \& Cognition, 18, 777-784.

RADVANSKY, G. A. (1998). The organization of information retrieved from situation models. Psychonomic Bulletin \& Review, 5, 283-289.

RadvanSKY, G. A. (1999). Memory retrieval and suppression: The inhibition of situation models. Journal of Experimental Psychology: General, 128, 563-579.

Radvansky, G. A., Gerard, L. D., Zacks, R. T., \& Hasher, L. (1990). Younger and older adults' use of mental models as representations for text materials. Psychology \& Aging, 5, 209-214.

Radvansky, G. A., Spieler, D. H., \& ZaCKS, R. T. (1993). Mental model organization. Journal of Experimental Psychology: Learning. Memorv, \& Cognition, 19, 95-114.

Radvansky, G. A., \& ZaCks, R. T. (1991). Mental models and the fan effect. Journal of Experimental Psychology: Learning, Memory, \& Cognition, 17, 940-953.

RADVANSKY, G. A., \& ZACKS, R. T. (1997). The retrieval of situationspecific information. In M. A. Conway (Ed.), Cognitive models of memory (pp. 173-213). Cambridge, MA: MIT Press.

RadVANSKy, G. A., ZACKS. R. T., \& HASher, L. (1996). Fact retrieval in younger and older adults: The role of mental models. Psychology \& Aging, 11, 258-271.

RiNCK, M., \& BowER. G. H. (1995). Anaphora resolution and the focus 
of attention in situation models. Journal of Memory \& Language, 34, $110-131$.

TAYLOR, H. A., \& TVERSKY, B. (1997). Indexing events in memory: Evidence for index dominance. Memory, $5,509-542$.

Trabasso, T., van den Broek, P., \& Suh, S. Y. (1989). Logical necessity and transitivity of causal relations in stories. Discourse Processes, 12, 1-25.

VAN DEN BROEK, P. W. (1988). The effects of causal relations and hierarchical position on the importance of story statements. Journal of Memory \& Language, 27, 1-22.

VAN DiJK, T. A., \& KINTSCH, W. (1983). Strategies in discourse comprehension. New York: Academic Press.

Wilson, S. G., Rinck, M., McNamara, T. P., Bower, G. H., \& MorROW, D. G. (1993). Mental models and narrative comprehension Some qualifications. Journal of Memory \& Language, 32, 141-154.
Zwaan, R. A., Langston, M. C., \& Graesser, A. C. (1995). The construction of situation models in narrative comprehension: An eventindexing model. Psychological Science, 6, 292-297.

Zwaan, R. A., Magliano. J. P., \& Graesser, A. C. (1995). Dimensions of situation model construction in narrative comprehension. Journal of Experimental Psychology: Learning, Memory, \& Cognition, 21, 386-397.

ZWAAN, R. A., \& RADVANSKY, G. A. (1998). Situation models in language comprehension and memory. Psychological Bulletin, 123, 162-185.

Zwaan, R. A., Radvansky, G. A., Hilliard, A. E., \& Curiel, J. M. (1998). Constructing multidimensional situation models during reading. Scientific Studies of Reading, 2, 199-220.

ZWAan, R. A., \& VAN OOSTENDORP, H. (1993). Do readers construct spatial representations in naturalistic story comprehension? Discourse Processes, 16, 125-143.

\section{APPENDIX A \\ Sample Story Used in the Experiment}

David walked along the banks of the river in town. Although the days were unbearably hot, the nights could be bitterly cold. He hugged his thin dust-covered coat around him as he thought about how the travel agent had lied to him. She had said that North African Sahara towns were friendly and romantic. Everything so far suggested the opposite.

A steady rain began to pour from the sky. (functional context)

The clouds were blocking out the moonlight, making the night darker. He needed some light so he could read his map. (nonfunctional context)

David was standing below an old bridge. (critical sentence)

He listened to the carts moving along the road as he took stock of their misfortunes thus far. The townsfolk treated you with contempt if you didn't speak the native Arabic or French. His wallet and passport had been stolen. Maureen and he were shocked to find out how decrepit and dirty their hotel was. Even the coffee they were served was bad. David was sure this trip would bring his troubled marriage to an end.

\section{APPENDIX B}

In Experiment $A, 36$ people read stories using only the functional context. There were four critical sentence versions, which were the four versions used in the recognition test of the main experiment. Reading times were faster for the functional ( $187 \mathrm{msec} / \mathrm{syllable}, S E=9)$ than for the nonfunctional-object ( $207 \mathrm{msec} /$ syllable, $S E=10)$, nonfunctional-spatial term ( $203 \mathrm{msec} /$ syllable, $S E=10$ ), and nonfunctionalboth (220 msec/syllable, $S E=13)$ versions. There was also higher recall of the critical spatial relation for the functional $(36 \%, S E=4)$ than the nonfunctional-object $(28 \%, S E=4)$, nonfunctional-spatial term $(23 \%, S E=4)$, and nonfunctional-both $(17 \%, S E=3)$ versions.

In Experiment $B$, recognition was used instead of recall. Thirty-six people were tested. Reading times were faster for the functional ( $176 \mathrm{msec} / \mathrm{syllable}, S E=7$ ) than the nonfunctional-object ( $193 \mathrm{msec} / \mathrm{syl}$ lable, $S E=10)$, nonfunctional-spatial term ( $200 \mathrm{msec} / \mathrm{syllable}, S E=8)$, and nonfunctional-both $(222 \mathrm{msec} / \mathrm{syllable}, S E=15)$ versions. Recognition was better for the functional $(86 \%, S E=2)$ than for the nonfunctional-object $(74 \%, S E=3)$, nonfunctional-spatial term $(77 \%, S E=3)$, and nonfunctionalboth $(73 \%, S E=4)$ versions.

In Experiment $C, 48$ people were tested using recall and recognition with the nonfunctional context of the main experiment. This reassigned the conditions, making one of the nonfunctional versions functional. Reading times were faster for the functional $(181 \mathrm{msec} / \mathrm{syllable}, S E=8)$ than for the nonfunctional-object ( $203 \mathrm{msec} / \mathrm{syllable}, S E=10)$, nonfunctional-spatial term ( $197 \mathrm{msec} / \mathrm{syllable}, S E=9)$, and nonfunctional-both (199 msec/ syllable, $S E=9)$ versions. Also, recall was higher for the functional $(44 \%, S E=3)$ than for the nonfunctional-object $(30 \%, S E=4)$, nonfunctional-spatial term $(35 \%$, $S E=4)$, and nonfunctional-both $(36 \%, S E=4)$ versions. Finally, recognition was better for the functional $(83 \%, S E=3)$ than for the nonfunctional-object $(74 \%, S E=3)$, nonfunctional-spatial term $(67 \%$, $S E=3)$, and nonfunctional-both $(71 \%, S E=4)$ versions. 